\title{
Semiclassical theory of the quantum Hall effect
}

\author{
F. Evers \\ Institut für Theorie der Kondensierten Materie, Universität Karlsruhe, Kaiserstrasse 12, 76128 Karlsruhe, Germany \\ W. Brenig \\ Department für Physik, Technische Universität München, D-85747 Garching, Germany
}

(Received 15 August 1997)

\begin{abstract}
The localization-delocalization transition occurring in the quantum Hall effect is studied for noninteracting, spinless electrons subject to a disordered long-range potential. Within the Chalker-Coddington network we compute the localization length at various energies and for a wide distribution of disorder strengths $W$. A scaling analysis with respect to the system size and the disorder reveals a crossover from quantum-mechanical to classical behavior that can be studied by means of a length scale $\xi_{\text {irr }}(W)$ which is "irrelevant" in the usual field-theoretical sense. We show that pronounced classical structures arise at parameter values $W$ an order of magnitude below the classical limit. They are stabilized by interference effects and give rise to $\xi_{\text {irr }} \propto W^{4 / 3}$, whereas for the localization length we still find $\xi(\epsilon) \propto \epsilon^{\nu}$ with $\nu \approx \frac{7}{3}$ in the entire investigated scaling regime. By relating our observations to recent results on the dynamical conductivity, we propose that the irrelevant scale actually leads to a "long-time tail" of $\sigma_{x x}(\omega)$ in long-range potentials. [S0163-1829(98)00303-8]
\end{abstract}

\section{INTRODUCTION}

It is a common belief now that the integer quantum Hall effect can be understood in terms of the localization scenario. ${ }^{1,2}$ The states at all energies except but one in the center of a disorder broadened Landau level are localized. A localization-delocalization transition takes place when the Fermi energy sweeping through a Landau band crosses its center.

In the case of noninteracting electrons, there have been several attempts to clarify how the localization of the twodimensional electronic states is destroyed by applying strong magnetic fields. The most intuitive approach is possible when the potential correlation length $d$ exceeds the magnetic length $\ell$ considerably. ${ }^{3}$ In this case the electron "feels", an electric field which can be considered as locally constant. It moves on equipotential lines, i.e., on contours $V(\vec{r})=\epsilon$. In the tails of the Landau band, the electronic states are localized as the corresponding electronic trajectories are closed. When the Fermi energy approaches the center of a band the electronic orbits are still closed, but they become larger and eventually extended at criticality. The correlation length $\xi_{p}$ $\propto\left|\epsilon-\epsilon_{c}\right|^{-\nu_{p}}$ diverges, and the critical exponent known from analogy to percolation theory is $\nu_{p}=\frac{4}{3}$. Semiclassical quantization restricts the number of allowed electronic trajectories, as the flux penetrating the area between two adjacent closed orbits has to be a multiple of the flux quantum.

It is only near "essential"' saddle points where this semiclassical picture breaks down because tunneling couples adjacent trajectories and induces a percolation network. However, as the tunneling probability is proportional to the overlap of the wave function $\exp \left[-(d / \ell)^{2}\right]$, in the limit $\ell / d \rightarrow 0$, tunneling becomes impossible, and the semiclassical model is valid.

A controversial argument by Milnikov and Sokolov incorporated the effect of tunneling at saddle points into this pic- ture, and related the classical percolation exponent $\nu_{p}$ and the full quantum-mechanical exponent $\nu_{\mathrm{qm}}$ for the localization length. ${ }^{4,5}$

An electronic trajectory with extension $r$ at energy $\epsilon$ from the band center crosses a number $n(\epsilon)=r / \xi_{p}(\epsilon)$ of "essential" saddle points. Since the transfer probability along the classical trajectory from one saddle point to the next equals unity, the corresponding probability amplitude for the trajectory is dominated by the overlap of the wave function near the saddle points: $\exp \left[-n(\epsilon)(d / \ell)^{2}\right]$. Assuming a parabolic approximation $d^{2} \propto \epsilon$, one immediately arrives at $\nu_{\mathrm{qm}}=\nu_{p}+1=\frac{7}{3}$. This result is in perfect agreement with numerical calculations and experiments. ${ }^{1}$

There are at least two crucial assumptions underlying the foregoing argument: Strictly speaking, the tunneling probability is proportional to the overlap of the wave function only if it is sufficiently small. Furthermore, it is implied that objects with an extension $\xi_{p}$ couple weakly essentially through a single saddle point. If the energy is in the vicinity of the band center, both approximations become questionable.

In the first part of our paper, following the ideas of Milnikov and Sokolov, we incorporate tunneling in a random walk model for the classical trajectories suggested by Wysokinski, Evers, and Brenig. We study numerically how the mean extension of the paths varies with energy. We find that even small tunneling probabilities destroy the localized character of the classical paths in a broad range of energies near the band center. In particular, we have been unable to observe a transition region exhibiting exponentially localized states with a corresponding exponent $\nu=\frac{7}{3}$, as predicted by the argument given above. This type of percolation picture does not capture the relevant aspects of the localization physics in the quantum Hall effect.

In the considerations leading to $\nu=\frac{7}{3}$ interference effects are unimportant. However, Chalker and Coddington ${ }^{6}$ showed that taking random phases into account one can reproduce 
$\nu_{\mathrm{qm}}=2.5 \pm 0.5$ even with all saddle points at the same energy. In their network model the critical behavior is due to phase fluctuations ${ }^{7}$ brought about by the spatial disorder in the position of the saddle points rather than by their fluctuations in energy. The latter constitute an irrelevant perturbation for the critical behavior. ${ }^{8}$ The classical limit is attained only for an infinite magnitude of the potential fluctuations.

Lee, Wang, and Kivelson ${ }^{9}$ used generalizations of the network model of Ref. 6, including fluctuating tunneling probabilities at the saddle points to study the crossover from quantum to percolation-type behavior: One expects to find a numerical value for the localization length exponent $\nu$ close to 2.3 for weak potential fluctuations similar to the earlier result of Ref. 6. With increasing fluctuation strength, the regime of energies near the band center where one obtains this quantum behavior should shrink, and for energies much larger than the tunnel width of a typical saddle point, one should find a classical regime where $\nu$ is close to $\frac{4}{3}$.

In this strict sense Lee, Wang, and Kivelson did not observe a crossover. In the particular network realization, where they observe a quantum type of behavior, they found it at all energies within the entire range of the numerically accessible disorder strength. To see the classical exponent, they enforced the classical limit by qualitatively changing the distribution function of the saddle-point energies: In the quantum case it is homogeneous in $[-W, W]$ and zero elsewhere, whereas in the classical case it consists of two delta functions, $\delta(\epsilon-W)$ and $\delta(\epsilon+W) .{ }^{10}$ This model exhibits only classical behavior, and no quantum behavior within the system sizes considered.

From our results of the random-walk model, we know that classical trajectories are extremely unstable with respect to tunneling. We show, in Sec. II of our paper, that localization in the network model depends crucially on interference, even if one observes the classical exponent $\frac{4}{3}$. We do so by demonstrating that, for given values of disorder strength and energy, the localization length can be finite or infinite (more precisely, much larger than the analyzed system sizes) depending on the rules for the phase shifts in the model. We conclude that interference effects suppress tunneling at saddle points so efficiently that observables can expose classical features like $\nu=\frac{4}{3}$ even if the potential fluctuations are at least an order of magnitude below the true classical limit.

This result is of particular interest, as in recent work ${ }^{11,12}$ on the dynamical conductivity in the quantum Hall effect the classical long-time tail anomaly ${ }^{13}$ has been found in two different quantum calculations. Classical physics alone cannot explain this result because the conductivity calculations have been done in a physical model potential with a homogenous energy distribution function with finite width and a potential small correlation length $d=2 \ell$.

In Sec. III of our paper, we suggest a solution for this problem and reexamine the crossover from classical to quantum percolation. We study the localization length using Chalker and Coddington's network model in the version of Eastmond and Chalker ${ }^{14}$ in the entire numerically accessible range for the disorder fluctuations up to $W=30$. As Lee, Wang, and Kivelson before, we failed to see the crossover in the localization length directly. Since disorder turns out to be irrelevant for the critical behavior, it is natural to study its effect on the leading irrelevant length $\xi_{\text {irr }}$. It describes how
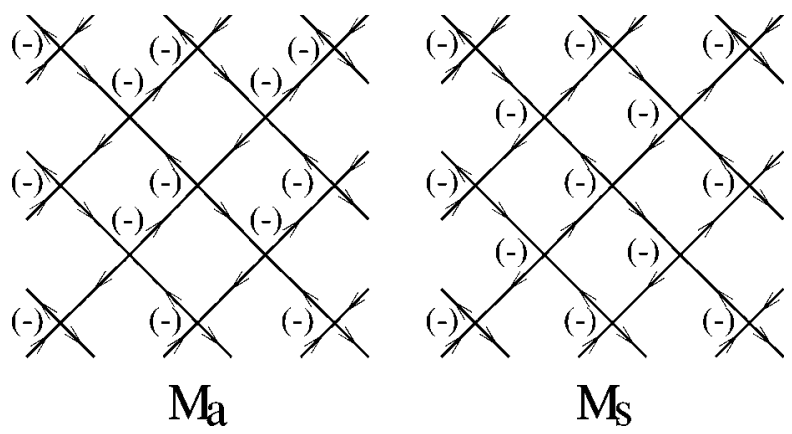

FIG. 1. Map of the lattice constituting the network. The arrangement of systematic phase shifts in models $\mathbf{M}_{\mathbf{a}}$ and $\mathbf{M}_{\mathbf{s}}$ explained in Sec. III B, is indicated for later reference.

node disorder drives the system away from the critical point, and is defined in Eq. (4). On the basis of a physical interpretation of this scale, we propose that in the quantum limit $W \rightarrow 0$,

$$
\xi_{\text {irr }} \propto\left(\frac{W}{\Delta}\right)^{1 / y_{\text {irr }}}, \quad y_{\text {irr }}=\frac{D_{2}}{2}-\frac{1}{\nu_{\text {qm }}}=0.35 \pm 0.05,
$$

whereas, in the classical limit $W \rightarrow \infty$,

$$
\xi_{\text {irr }} \propto\left(\frac{W}{\Delta}\right)^{\nu_{p}}, \quad \nu_{p}=\frac{4}{3} .
$$

Here $\Delta$ is an energy scale denoting the tunnel width of a saddle point, and $D_{2}$ is the correlation dimension. ${ }^{1}$ Our numerical data support this crossover scenario. Since the frequency dependence of the conductivity at criticality is not universal, we conclude that the observation of the classical long-time tail in full quantum calculations should be related to the crossover in the irrelevant scale.

\section{A CLASSICAL LATTICE MODEL PLUS TUNNELING}

Only recently, a model was introduced which allows us to simulate the motion of classical particles under quantum Hall conditions in an efficient manner. This model is explained in detail elsewhere, ${ }^{15}$ so we restrict ourselves to a few basic notions.

We model classical trajectories in a random potential by a lattice model. The nodes correspond to the saddle points, whereas the links represent the classical paths. The energy distribution of the nodes is taken to be homogeneous in the interval $[-W, W]$. As indicated in Fig. 1, we attach arrows to the links, taking the chiral nature of the trajectories into account.

An ant, traveling on the lattice, carries an energy $\epsilon$. When arriving at a saddle point, it first measures its energy $V_{\mathrm{sp}}$. Then it calculates the "tunneling probability" according to

$$
T(\epsilon)=\frac{1}{1+\exp \left(\epsilon-V_{\mathrm{sp}}\right)} .
$$

It turns right (left) with probability $T(1-T)$. For instance, in the classical lattice model considered in Ref. 15, $T$ is either 0 or 1.

The result for $T$, due to Fertig and Halperin, ${ }^{16}$ is valid only if the trajectories involved are extended, whereas typi- 


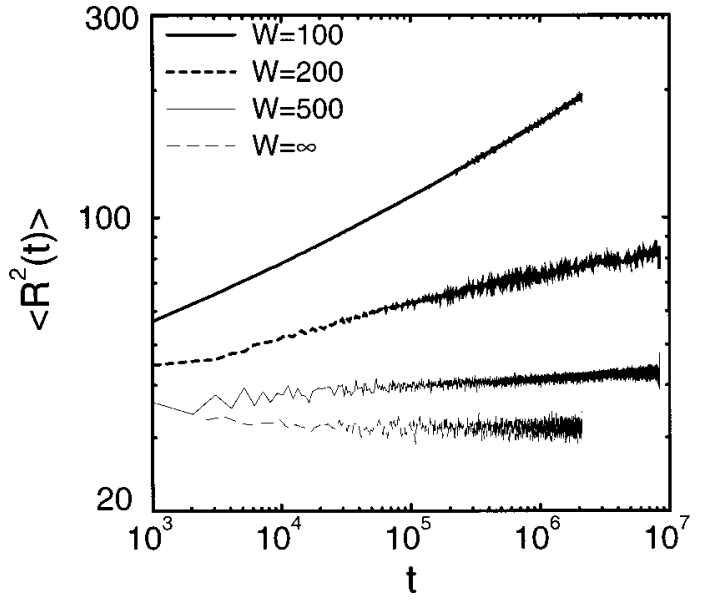

FIG. 2. Mean-square displacement of ant tracks on lattices with varying disorder strengths $W$. The ratio $\epsilon / W=0.2$ is fixed. Every curve was obtained after averaging 1000 paths.

cal equipotentials in a random potential are closed, so that interference effects become significant. These are neglected in the current approach. For $|\epsilon|>V_{\mathrm{sp}}$ we recover the correct expression for the tunneling probability as used above.

The model is constructed such that the presumptions underlying the semiclassical argument are fulfilled. In the limit of large $W$, the ant walks on classical paths, which are coupled only via saddle points with a tiny tunneling probability $T$.

We calculate the mean-square deviation of the ant's path $\left\langle R_{\epsilon}^{2}(t)\right\rangle$ at various energies $\epsilon$. If exponential localization holds true, one expects $\left\langle R_{\epsilon}^{2}(t \rightarrow \infty)\right\rangle$ to have an upper boundary proportional to $\xi^{2}(\epsilon)$. Indeed, taking the classical limit $W \rightarrow \infty$ with a typical ratio $\epsilon / W=0.2$ fixed, the mean-square deviation attains a finite value. In Fig. 2 this limit is represented by the lowest curve, which is basically independent of time. However, at huge but finite values of the disorder, $\left\langle R^{2}(t)\right\rangle$ does not saturate.

The existence of a finite minimal tunneling probability $T_{\min }$ on the lattice gives rise to a lower boundary of the diffusion coefficient $D_{\min }=d^{2} T_{\min }$, where $d$ denotes the lattice constant. Therefore, at finite $W$, we expect the ants to be delocalized regardless of their energy. This, however, cannot be the reason for the pronounced divergence of $\left\langle R^{2}(t)\right\rangle$. First, we note, that $D_{\text {min }} \approx e^{-\mathrm{W}}$, e.g., is practically zero. Second, the mechanism under consideration would imply that time scales with $T_{\min }$ so that in a double-logarithmic plot all curves are more or less parallel, which obviously is not the case.

One gains some insight by realizing that along a classical $n$-step trajectory at $\epsilon \ll W$, there are approximately $n / W$ saddle points with a tunneling probability of order 1 . So, in general, a large classical orbit couples over many saddle points to other orbits, which gives rise to the strong though subdiffusive divergence we observe. The small but finite minimal tunneling probability $T_{\min }$ manifests itself in a small positive curvature in all data curves.

In contrast, the semiclassical argument assumes tacitly that there is one single saddle point yielding the dominant contribution. Our result demonstrates that the concept "classical percolation plus tunneling" fails to explain the localization scenario.

\section{CRITICAL BEHAVIOR AND INTERFERENCE}

To analyze interference effects on the localization properties of wave functions, we attach random-phase factors to the links of the lattice model. They account for the AharonovBohm factors connected with fluctuating spatial distances of the saddle points. Doing so, we essentially end up with a network model first introduced by Chalker and Coddington and later generalized by Eastmond.

The technical details are described in the literature ${ }^{6}$ and once again we restrict ourselves to a few remarks. To properly account for tunneling at the saddle points, we characterize the nodes by $2 \times 2$ transfer matrices,

$$
\mathbf{M}=\left(\begin{array}{cc}
e^{i \varphi_{1}} & 0 \\
0 & e^{i \varphi_{2}}
\end{array}\right)\left(\begin{array}{cc}
\sqrt{1+e^{-\pi \gamma}} & -\sqrt{e^{-\pi \gamma}} \\
-\sqrt{e^{-\pi \gamma}} & \sqrt{1+e^{-\pi \gamma}}
\end{array}\right)\left(\begin{array}{cc}
e^{i \varphi_{3}} & 0 \\
0 & e^{i \varphi_{4}}
\end{array}\right),
$$

where $\gamma=\epsilon-V_{\mathrm{sp}}$. The corresponding tunneling probability is compatible with Eq. (1). The diagonal matrices are meant to incorporate the phase factors along the links between two nodes. Disorder can be introduced into the model by choosing at random phases from the interval $[0,2 \pi]$ and the energy of the saddle point $V_{\text {sp }}$ from $[-W, W]$ as before. We apply periodic boundary conditions and calculate the eigenvalues of the transfer matrix on long cylinders of length $N$ nodes and width $M$ nodes. The localization length is

$$
\xi_{\mathrm{M}}=\frac{1}{4 N \ln \left(\gamma_{M / 2}\right)},
$$

where $\gamma_{M / 2}$ denotes the eigenvalue of the transfer matrix closest to 1 . We take $N=1.28 \times 10^{6}$. The result has to be extrapolated to $M \rightarrow \infty$.

A considerable amount of work has already been done on this model ${ }^{6,14,9}$ for much smaller values of the disorder $W .{ }^{17}$ All authors agree that $\nu_{\mathrm{qm}}=2.3 \pm 0.1$. Another universal number is the value $\Lambda_{c}$ of the renormalized localization length

$$
\Lambda(\epsilon)=\frac{\xi_{M}(\epsilon)}{M}
$$

at $\epsilon=\epsilon_{c}$ in the limit $M \rightarrow \infty$. Whereas most authors agree with the original result obtained by Eastmond,

$$
\Lambda_{c}=1.21 \pm 0.01
$$

Lee, Wang, and Kivelson differ from this value by $10 \%$.

\section{A. Network model with random-phase shifts}

We display our results for the scaling function of the renormalized localization length $\Lambda_{M}(\epsilon)$ in systems with large disorder $W=30$ in Fig. 3, with filled symbols. Because of rounding errors, we could not go to higher values of $W$. If scaling worked well, all data should collapse onto a single curve. They tend to do so for the largest system sizes, which correspond to the uppermost curves. For smaller systems, the data scale only in the high-energy tail, where the localization length is sufficiently small for finite-size effects to be negligible. This leads to an estimate for the exponent $\nu=2.3$ shown in the inset. Our result is consistent with the conjec- 


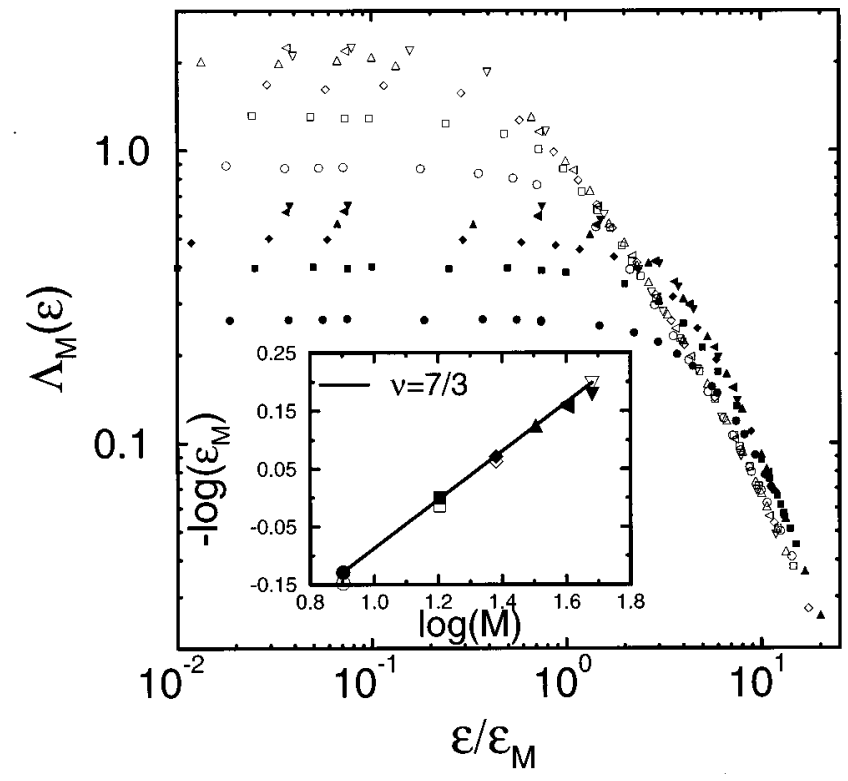

FIG. 3. Scaling function of the localization length in systems of width $M=8,16,24,32,40$, and 48 at very large disorder $W=30$ in a model with random, physical $\left(M_{r}\right.$, filled symbols) and systematical, symmetrical ( $M_{s}$, empty symbols) phase shifts. The lowest curve (circles) denotes the smallest system size, the uppermost curve (triangle down) the largest system size. The statistical and numerical errors are not larger than the symbol size. Inset: Scaling factor $\epsilon_{M}$ resulting from rescaling of the energy axis. The solid line is not a fit, but is meant for comparison. The logarithm has base 10 .

ture of other authors, stating that potential fluctuations give rise only to an irrelevant scaling field. ${ }^{14,8,19}$

\section{B. Network models with non-random-phase shifts}

Introducing interference restored the critical properties of the otherwise noncritical lattice model. Next we ask how these properties change if we vary the rules for the phase factors along the links.

Modifying the phase shifts is easily accomplished in the network model, as in Eq. (2). It allows for different arrangements of the systematic phase shifts such that one ends up with the original Chalker-Coddington model when introducing phase disorder. We study two cases $\left(\mathbf{M}_{\mathbf{s}}\right.$ and $\left.\mathbf{M}_{\mathbf{a}}\right)$ in which all random-phase factors vanish: $\varphi_{i}=0$. The remaining systematic phase shifts are organized as depicted in Fig. 18

In Fig. 3 we confront the results of a scaling analysis of the physical case of $\varphi_{\mathrm{i}}$ chosen completely at random as discussed above (model $\mathbf{M}_{\mathbf{r}}$, filled symbols; and model $\mathbf{M}_{\mathbf{s}}$, empty symbols). The latter was analyzed by Lee in the limit of small disorder. ${ }^{19} \mathrm{He}$ found that $\nu=1$, and that small disorder is an irrelevant perturbation at this fixed point. However, we are interested in the other limit of large disorder, and observe a similar value $\nu \approx 2.3$ in the numerically accessible intervall of system sizes $8 \leqslant M \leqslant 48$ for both models.

We also calculated the renormalized localization length in model $\mathbf{M}_{\mathbf{a}}$. In contrast to the previous case of model $\mathbf{M}_{\mathbf{s}}$, here we find no hint of localization at all up to the system size $M=64$, as can be seen in Fig. 4 .

At this point two questions deserve discussion: (1) Why do models $\mathbf{M}_{\mathbf{r}}$ and $\mathbf{M}_{\mathbf{s}}$ exhibit localization in a certain range

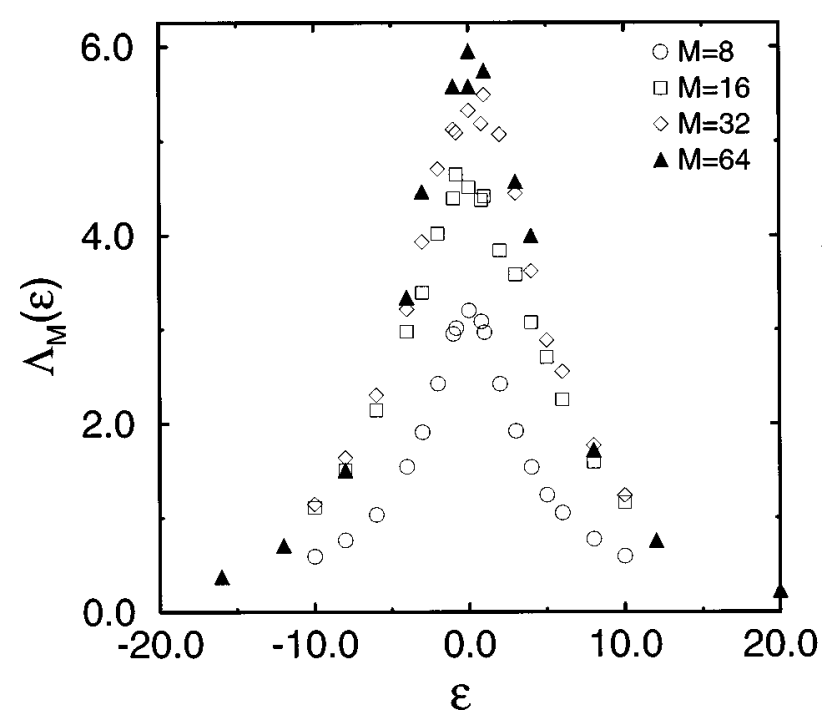

FIG. 4. Renormalized localization length in model $\mathbf{M}_{\mathbf{a}}$. Localization is indicated by the intersection of all curves in a single point. The statistical errors are considerably larger than the symbol size, and can be read from the scattering of the data at zero energy.

of system sizes, while model $\mathbf{M}_{\mathbf{a}}$ does not? Figure 1 displays the arrangement of the $\pi$ phase shifts denoted by the minus signs in the transfer matrix on the lattice. One can easily convince oneself that a closed loop in model $\mathbf{M}_{\mathbf{s}}$ always comes with a phase factor of -1 .

In contrast, in model $\mathbf{M}_{\mathbf{a}}$ phase factors -1 and 1 can occur. Therefore, we believe that localization in model $\mathbf{M}_{\mathbf{s}}$, and also in $\mathbf{M}_{\mathbf{r}}$, occurs because of suppression of tunneling due to destructive interference. In other words, the tunnel splitting induced by the coupling of two closed classical orbits can be greatly reduced because of phase effects.

(2) Is large disorder relevant in model $\mathbf{M}_{\mathbf{s}}$ ? To answer this question we have repeated the procedure leading to Fig. 3 with slightly smaller values for the disorder. The results are depicted in Fig. 5. The curves are no longer linear, but have a slope increasing with the system size. Since the slope de-

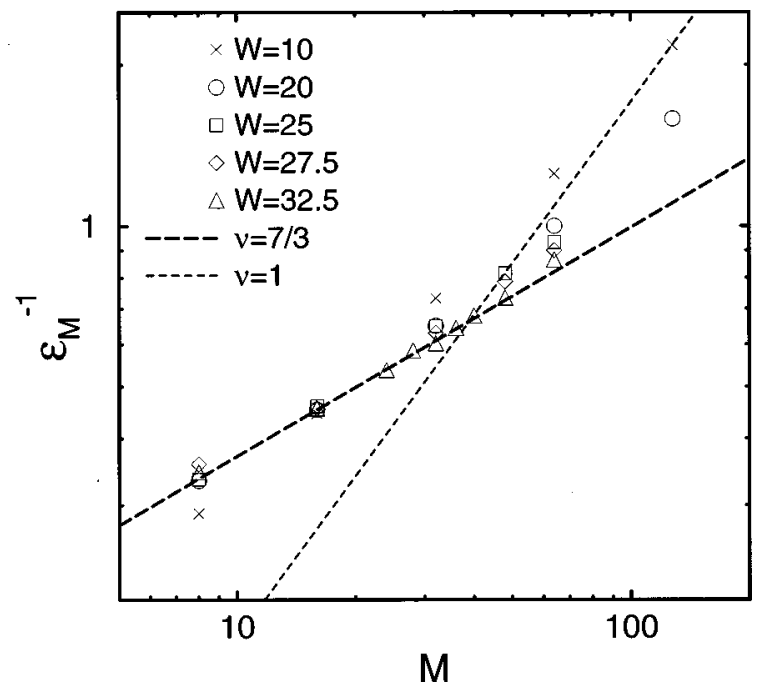

FIG. 5. Similar data as depicted in the inset of Fig. 3 for model $\mathbf{M}_{\mathbf{s}}$, though now also for smaller values of the disorder parameter $W$. The broken lines are meant for orientation. 


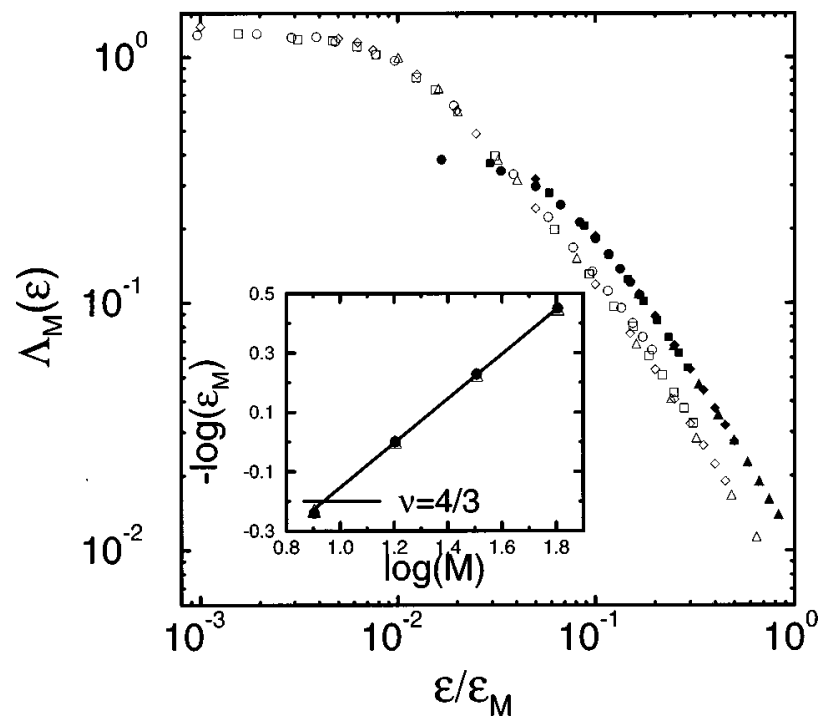

FIG. 6. Data similar to Fig. 3. Here, however, the saddle-point energies are taken to be either $-W$ or $W$ only $(W=4.2)$.

fines $1 / \nu$, we expect $\nu$ to decline to 1 in the thermodynamic limit. Hence large disorder should also be irrelevant in model $\mathbf{M}_{\mathrm{s}} \cdot{ }^{21}$

The absence of localization in systems $\mathbf{M}_{\mathbf{a}}$ with an asymmetric choice of phase shifts remains even in models exhibiting the classical exponent $\frac{4}{3}$ when choosing the symmetric versions $\mathbf{M}_{\mathrm{s}}$ or $\mathbf{M}_{\mathbf{r}}$. To see this, we have repeated the calculations for the three models $\mathbf{M}_{\mathrm{r}}, \mathbf{M}_{\mathrm{s}}$, and $\mathbf{M}_{\mathrm{a}}$ but using a different distribution function for the saddle-point energies. Whereas previously we picked $V_{\mathrm{sp}}$ at random from the interval $[-W, W]$, we now choose it to be $-W$ or $W$ only. Taking $W$ as large as possible, we try to reduce the coupling between the classical paths. For models $\mathbf{M}_{\mathbf{r}}$ and $\mathbf{M}_{\mathrm{s}}$, scaling works reasonably well (Fig. 6), and we obtain the classical value $\nu=\frac{4}{3}$ for the localization length exponent. Once again the model $\mathbf{M}_{\mathbf{a}}$ with the asymmetric systematic phase shifts does not show any sign of localization within the analyzed system sizes (Fig. 7). Thus it is suggested that the tunnel

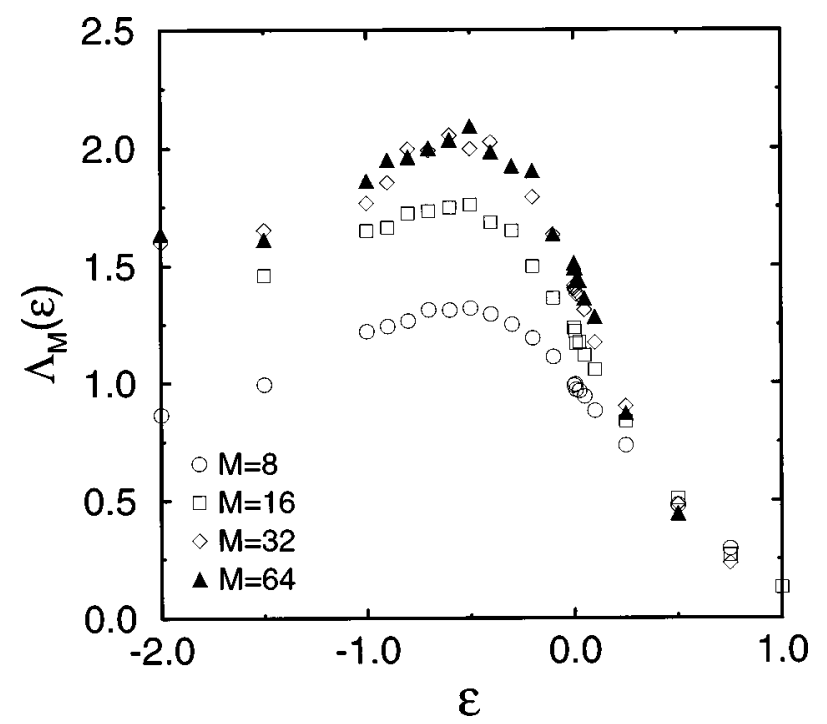

FIG. 7. Data similar to Fig. 4. However, the saddle-point energies are taken to be $-W$ or $W$ only $(W=4.2)$.

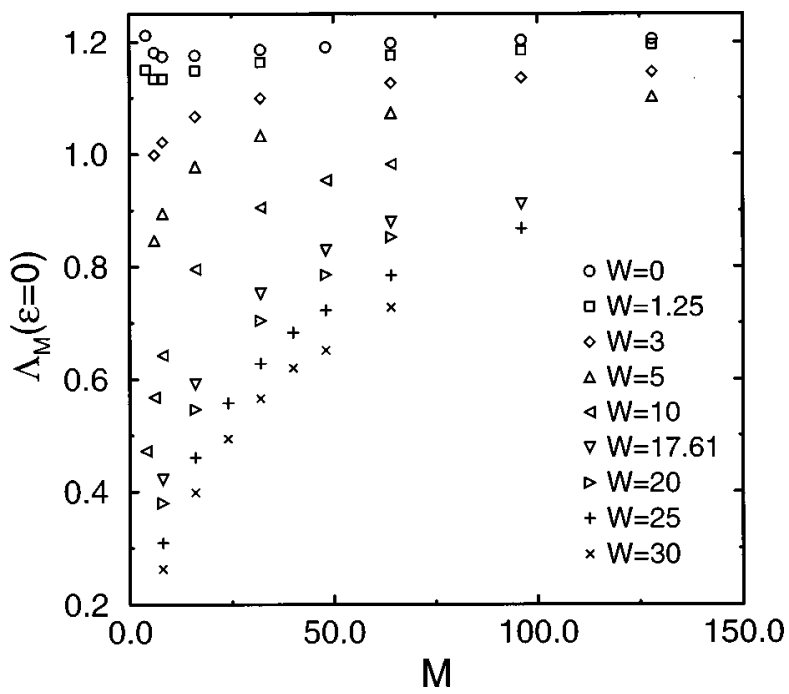

FIG. 8. Renormalized localization length $\Lambda_{M}(0)=\xi_{M}(0) / M$ for nine different disorder strengths $W$. The statistical errors are smaller than the symbol size.

splitting can eventually vanish. This is analytically verified for tractable model potentials. ${ }^{20}$

\section{IV. "IRRELEVANT LENGTH SCALE" AND CROSSOVER SCENARIO}

If one compares the magnitudes of the disorder applied in the random-walk model, and the network model one arrives at the conclusion that the maximal disorder in the latter is at least an order of magnitude below the threshold to the classical limit, where tunneling is suppressed by potential fluctuations. Thus it seems unlikely to observe the crossover from the quantum exponent 2.3 to the classical exponent $\frac{4}{3}$ directly within the given framework.

On the other hand, we have seen that potential fluctuations have a strong impact on the finite-size scaling properties. When turning on the disorder, one has to go to larger system sizes to see the scaling behavior. Thus it seems natural to study how much the system size needs to be increased.

At the critical point $\left(\epsilon=\epsilon_{c}\right)$, one expects the localization length to obey a scaling law

$$
\xi_{M}\left(\epsilon_{c}\right)=M \Lambda\left(g_{1} M^{-y_{1}}, g_{2} M^{-y_{2}}, \ldots\right) .
$$

Here, $g_{1}, g_{2} \ldots$ denote the coupling constants to the leading irrelevant scaling fields, and $y_{1}, y_{2} \ldots$ the corresponding scaling indices. As a definition of the leading irrelevant length scale we choose

$$
\xi_{\text {irr }}=g_{1}(W)^{1 / y_{\text {irr }}} .
$$

The ratio of this scale to the system size $M / \xi_{\text {irr }}$ is a measure of how well finite-size scaling works. It defines an effective system size.

Next we perform a finite-size analysis of our data displayed in Fig. 8. To this end we expand the scaling function and keep only the leading finite-size correction

$$
\Lambda_{M}\left(\epsilon_{c}\right)=\Lambda_{c}-g_{1}(W) M^{-y_{\text {irr }}} .
$$

In principle, one could continue by fitting all data simultaneously according to this expression. It turns out, though, 


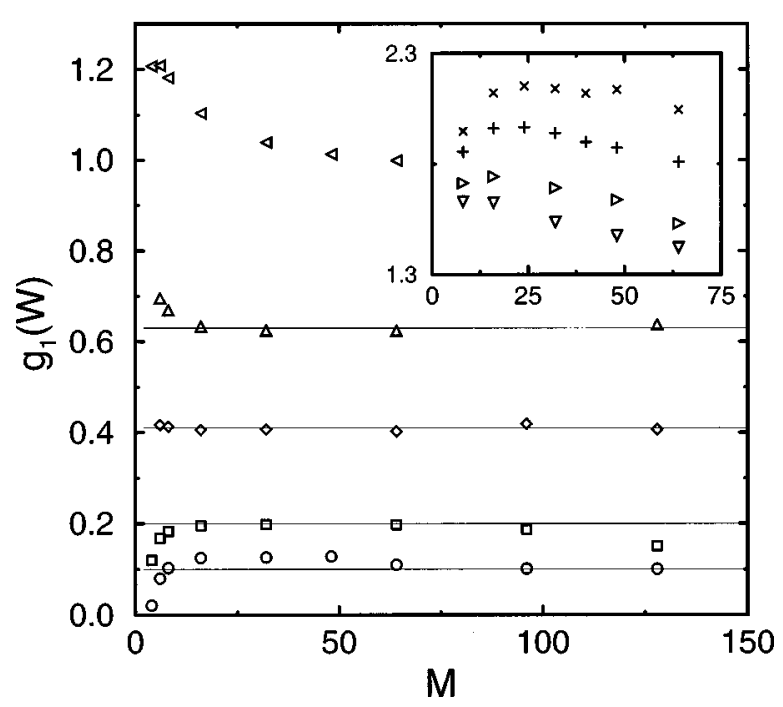

FIG. 9. Data from Fig. 8 after subtracting $\Lambda_{c}=1.225$ and then multiplying by $M^{y_{\text {irr }}}$ with $y_{\text {irr }}=0.34$. The inset shows the result for disorder $W>10$.

that given our system sizes the contribution of the higherorder terms is important for disorder $W>5$. It is more reliable to assume a value for $\Lambda_{c}$, subtract this number from the scaling function, and multiply the difference with $M^{y_{\text {irr }}}$. The result of this procedure is shown in Fig. 9. We estimate $\Lambda_{c}=1.22 \pm 0.01$ and $y_{\text {irr }}=0.35 \pm 0.05$, in agreement with Eastmond, who observed $1.21 \pm 0.02$. The error bars in $y_{\text {irr }}$ and $g_{1}$ are mainly a consequence of the uncertainty in $\Lambda_{c}$.

The scaling of the data with $W<10$ works reasonably well. For larger disorder, however, our system sizes are too small in order to extract $g_{1}$. Without further extrapolation we obtain only an upper limit for $g_{1}$. Therefore we have ignored these data when extracting $\Lambda_{c}$ and $y_{\mathrm{irr}}$.

Finally, Fig. 10 displays the outcome for the coupling constant $g_{1}(W)$. There are two different regimes $W \rightarrow 0$ and $W \rightarrow \infty$ characterized by power laws with different exponents. In what follows, we argue that these exponents can be related to $y_{\text {irr }}$ and $\nu_{p}$.

\section{1. $W \rightarrow 0$}

We consider the limit of vanishing disorder first. In our model, fluctuations in the energy of the saddle points are equivalent to energy fluctuations along the trajectory. Thus the effect of the node disorder can be interpreted as a coupling of the electron to an external spatially fluctuating field. The coupling constant is $W$. One expects the scaling function $\Lambda(W)$ to be linear in $W$ in the limit $W \rightarrow 0 .^{22}$ Therefore, it seems plausible that $g_{1}$ has an expansion

$$
g_{1}(W)=c_{1}\left(W_{0}+W\right)+O\left(W^{2}\right)
$$

where $W_{\text {eff }}=W_{0}+W$ defines an effective node disorder. We estimate $W_{0}=1.0 \pm 1.0$ and $c_{1}=0.08 \pm 0.05$ from our numerical data.

With the definition $\xi_{\text {irr }} \propto W_{\text {eff }}^{\nu^{\prime}}$, we can identify $\nu^{\prime}=1 / y_{\text {irr }}$. On the other hand, on physical grounds it seems plausible that the irrelevant length arises from small spatial fluctua-

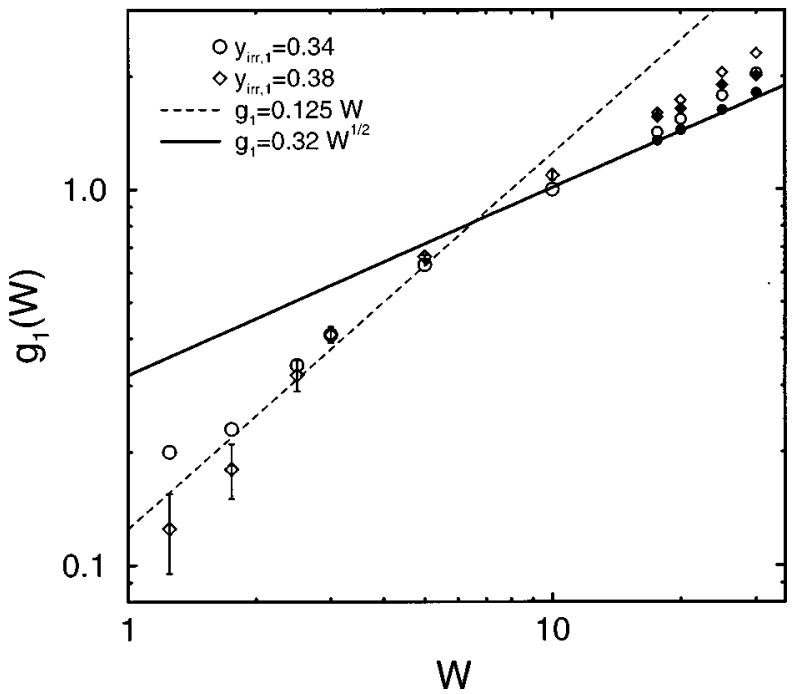

FIG. 10. Coupling constant $g_{1}(W)$ to the leading irrelevant scaling operator as obtained from Fig. 8. We have depicted results for $g_{1}$ when choosing $\Lambda_{c}=1.21$ (1.225) and $y_{\text {irr }}=0.38$ (0.34) for comparison. The empty symbols for $W>10$ give as an upper limit $g_{1}$ as estimated from a system size $M=64$. This procedure leads to an upper bound for the exponent $x$. In order to obtain a somewhat improved estimate, we have extrapolated our data. The full symbols denote the estimated result using a simple extrapolation scheme: we have assumed that the data in the interval $10 \leqslant W \leqslant 30$ drop by the same fraction from their maximum value when $M \rightarrow \infty$. The dashed and solid lines are given for orientation.

tions in $\epsilon_{c}$. In the Appendix we propose a heuristic argument, similar to the derivation of the Harris criterion, ${ }^{23}$ that leads to the conjecture

$$
y_{\text {irr }}=\frac{D_{2}}{2}-\frac{1}{\nu_{\text {qm }}} \approx 0.35 \pm 0.05,
$$

which is confirmed by our numerical findings. $D_{2}$ denotes the "correlation dimension" of the wave function known from the multifractal analysis.

$$
\text { 2. } W \rightarrow \infty
$$

In the large disorder limit, the slope of $g_{1}(W)$ decreases from 1 to $\frac{1}{2}$, roughly. Therefore the exponent $\nu^{\prime}$ comes down from $2.75 \pm 0.3$ to values below 1.6.

In this limit, we expect the tunnel width at most saddle points to be very small due to interference, so that classicallike substructures can form. Their extension should be indicated by $\xi_{\text {irr }}$, and is proportional to $(\Delta / W)^{-4 / 3}$, where $\Delta$ denotes the typical tunnel width of those saddle points which actually provide the coupling of the classical substructures. Therefore we propose $\nu^{\prime}=4 / 3$. Klesse and Metzler observed similar semiclassical structures in their version of the network model. ${ }^{8}$ In particular, they recently confirmed our conjecture for the exponent $\nu^{\prime} .^{24}$

In our final numerical experiment, we report on another check of our interpretation of $\xi_{\text {irr }}$ as an effective underlying unit of length. The extension of electronic states sufficiently far in the tails of a Landau band is small, so that the corresponding localization length does not depend on the sample size. Scaling, however, is still valid, so that 


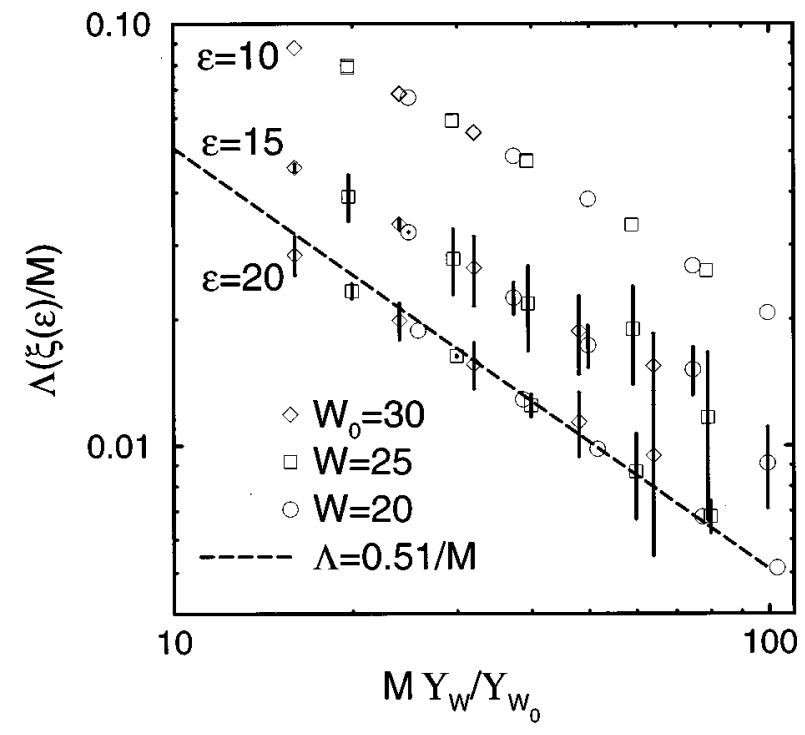

FIG. 11. Renormalized localization length for energies deep in the insulator. The localization length is almost independent of $M$ as $\Lambda_{M} \propto 1 / M$. Its dependence on the disorder can be eliminated by appropriate rescaling of the $M$ axis. The data have been shifted on the curve corresponding to $W_{0}=30$.

$$
\Lambda_{M}(\epsilon, W)=\frac{\xi\left(\epsilon, W_{0}\right)}{M} Y(W)
$$

Here, per definition, $\Lambda_{M}$ is proportional to $1 / M$ for energies characterizing states localized within the sample and also $Y\left(W_{0}\right)=1$. Assuming that our interpretation makes sense, the least to expect is that $Y(W)$ is proportional to $\xi_{\text {irr }}(W)$.

In Fig. 11 we depict $\Lambda_{M}$ for energies in the localized regime after rescaling the $M$ axis to extract $Y(W)$. The results are given in Fig. 12. We find

$$
Y(W) \propto W^{\nu^{\prime}}, \quad \nu^{\prime}=1.2 \pm 0.1
$$

Due to rounding errors it is difficult to go to higher values of $W$ which would be desirable to obtain more data points in a

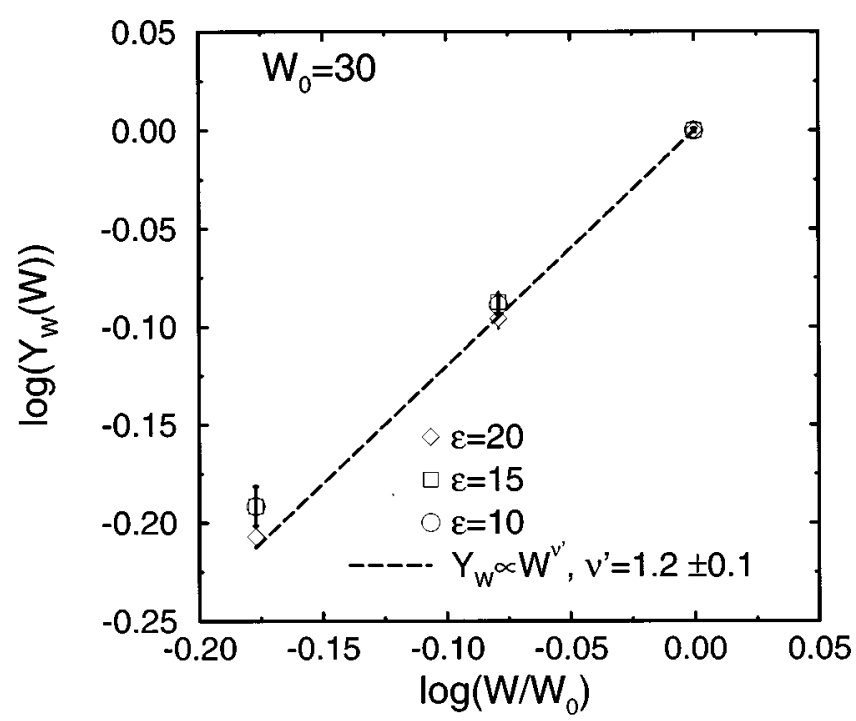

FIG. 12. Scaling factor $Y(W)$ from Fig. 11 as explained in the body of the text. wider range of $W$. However, the result is confirmed for three different energies. The deviation from the expected value $\nu^{\prime}=\frac{4}{3}=1.33$ is due to the fact that our energies are still not large enough to totally exclude finite-size effects.

Finally we relate our results to other recent experimental and numerical observations. Kratzer and Brenig, ${ }^{11}$ Gammel and Brenig, ${ }^{12}$ and the present authors ${ }^{13}$ computed the frequency-dependent conductivity under quantum Hall conditions using various different approaches. They found that $\sigma_{x x}(\omega)$ in short-range potentials $\ell / d \gg 1$ has conventional Drude-like features at wave vector $q=0$. Disregarding this trivial decay, it seems fair to say that at frequencies in the scaling regime $\sigma_{x x}$ is constant. In long-range potentials, however, one observes a long-time tail anomaly:

$$
\sigma_{x x}=\frac{e^{2}}{2 h}-\mathrm{const}|\omega|
$$

Assuming that universality for the conductivity holds, the frequency dependence of $\sigma_{x x}$ in the scaling regime should not hinge on the microscopic details of the underlying random potential like $d$ or $W$. Also, for long-range potentials we expect the leading behavior to be $\sigma_{x x}(\omega) \propto \omega^{0}$ in the scaling regime. ${ }^{25} \mathrm{We}$ consider particular features of the frequency behavior, namely, the long-time tails, as due to corrections to scaling. Hence we are led to the conclusion that the underlying classical substructure of the wave function, although in a field-theoretical sense irrelevant at the critical point, might dominate the dynamical response functions, and can be observed experimentally.

Experimental results by Dolgopolov et $a .^{26}$ might corroborate our conclusion: They measured the current-voltage characteristics in the vicinity of the localizationdelocalization transition in a temperature regime where the conductivity shows activated behavior. When analyzing their data, they introduced a length scale $L(\epsilon)$ as the ratio of the activation energy and the critical electric field where the $I-V$ characteristics becomes nonlinear. They observed $L$ $\propto\left|\epsilon-\epsilon_{c}\right|^{-\nu}$, with $\nu \approx 1$, and interpreted their result in terms of a classical percolation picture. Certainly, at zero temperature this interpretation breaks down, since tunneling is the only conduction mechanism, and phase coherence can no longer be neglected. Nevertheless, their example might demonstrate that experiments can show certain features that might find a proper interpretation in the classical percolation framework.

\section{SUMMARY}

First we concluded that the semiclassical argument as given by Milnikov and Sokolov does not provide a possible mechanism, leading to the divergence of the localization length with an exponent $\frac{7}{3}$ in the quantum Hall effect. By means of an appropriate numerical simulation, we explicitly demonstrated that taking its basic idea literally, namely, coupling of classical orbits at saddle points via tunneling and neglecting interference, one destroys the only localization mechanism in the model and thus obtains no finite localization length.

Nevertheless, we also demonstrated that classical features exist in Chalker and Coddington's network model at a disor- 
der strength an order of magnitude smaller than the values one would expect to be necessary from the model, ignoring phase fluctuations. As a consequence, we were able to study a crossover in the irrelevant length scale from the pure quantum limit into a regime where classical substructures already exist. The crossover is characterized by a change in the exponent $\nu^{\prime}$ in the power-law dependence of the irrelvant length scale on the disorder strength. Our analytical arguments for the numerical value of $\nu^{\prime}$ in both limits suggest, in particular, a relation between the leading irrelevant exponent $y_{\text {irr }}, D_{2}$, and $\nu$.

Finally, we related our findings to the recently discovered long-time tail anomaly in the frequency dependence of the diagonal conductivity for strong, long-range disorder potentials at zero wave number. We believe that this anomaly can, in principle, be understood in purely classical terms, and originates from the classical substructure of the wave functions. We argued that this substructure, although yielding wrong exponents $\nu$ and $\eta$ for the scaling behavior, nevertheless gives rise to the dominant frequency correction to the static value $\sigma_{x x}=e^{2} / 2 h$ of the conductivity.

\section{ACKNOWLEDGMENTS}

During the course of this work one of the authors (F.E.) appreciated useful discussions with more colleagues than can be listed here. Thus, while grateful to all of them, he mentions only John Chalker, for sending of a copy J. F. G. Eastmond's thesis, and R. Klesse, and his closest collaborators Dietrich Belitz, Berndt Gammel, Peter Kratzer, and Karol Wysokinski. He would particularly like to acknowledge the warm hospitality of the University of Oregon during the final stage of this work. This work was supported by NSF under Grant No. DMR-95-10185.

\section{APPENDIX}

Consider a $d$-dimensional network with size $L^{d}$ and small disorder characterized by an energy scale $W$. For a given realization of the disorder, one can calculate the Green's function $G(\epsilon, r)$. The energy $\epsilon_{c}$, at which $|G(\epsilon, L)|$ becomes maximal, fluctuates between different samples. Assuming that $\epsilon_{c}$ is the mean value averaged over subregions with fluctuating $\epsilon_{0}(\vec{r})$, the corresponding energy width is

$$
\Delta \epsilon_{c} \propto N^{-1 / 2} .
$$

$N$ denotes the number of saddle points or, equivalently, the area of the subregion contributing to the average. In connection with the Harris criterion, one usually assumes that averaging occurs over the whole sample, so that $N=L^{d}$.

A second energy interval is defined by

$$
\Delta \epsilon_{0} \propto L^{-1 / \nu} .
$$

It can be interpreted as the range of energies for which we expect electronic states extending throughout the sample if $W=0$. The Harris criterion states that disorder is irrelevant, if in the thermodynamic limit the ratio $r=\Delta \epsilon_{c} / \Delta \epsilon_{0} \ll 1$, or, equivalently,

$$
y=d / 2-1 / \nu>0 .
$$

It seems reasonable to assume that $\epsilon_{c} \propto W$ for small $W$. Thus the exponent $y$ controls how the system size must be rescaled after enlargening the disorder $W$ to restore the value for the ratio $r$. Hence we identify $y=y_{\text {irr }}$. Inserting $d=2$ and $1 / \nu \approx 0.43$, we arrive at $y_{\text {irr }}=0.57$, which is incompatible with our numerical findings $0.35 \pm 0.05$. We interpret this discrepancy as evidence that the assumption $N \propto L^{d}$ is, in general, wrong.

At least in the case of the integral quantum Hall effect, it is known that wave function correlations exist only in a subspace $L^{D_{2}}$ of the sample. In other words, amplitude fluctuations of the wave function between different regions in space are almost always uncorrelated. Fluctuations in the saddlepoint energies modify the correlation properties of the wave function only if they happen to occur in the subspace of the sample where long-range correlations exist. Therefore we propose a modified version of the Harris criterion in which the average is not taken over the entire sample but only over the subspace $L^{D_{2}}$. An argument similar in spirit was given earlier by Kramer. ${ }^{27}$ Hence the dimension $d$ of the support of the wave function should be substituted by $D_{2}$,

$$
y_{\text {irr }}=D_{2} / 2-1 / \nu \text {. }
$$

In principle, $D_{2}$ can be obtained from the multifractal analysis. Values between $D_{2}=1.51 \pm 0.03$ (Ref. 8) and $1.62 \pm 0.02$ (Ref. 28) have been published in the literature. We end up with the estimate $y_{\text {irr }}=0.35 \pm 0.05$, in agreement with the numerical result.
${ }^{1}$ M. Janssen, O. Viehweger, U. Fastenrath, and J. Hajdu, Introduction to the Theory of the Integer Quantum Hall Effect $(\mathrm{VCH}$, Weinheim, 1994).

${ }^{2}$ B. Huckestein, Phys. Rev. Lett. 72, 1080 (1994); Rev. Mod. Phys. 67, 357 (1995). The value for $\Lambda_{c}$ published in these works is 1.14, inconsistent with Eastmond's result $\Lambda_{c}=1.21$. In his most recent work, however, the author corrects his previous result and now agrees with Eastmond. We are grateful to B. Huckestein for communicating his findings.

${ }^{3}$ S. A. Trugman, Phys. Rev. B 27, 7539 (1983).

${ }^{4}$ G. V. Mil'nikov and I. M. Sokolov, Pis'ma Zh. Éksp. Teor. Fiz. 48, 494 (1988) [ JETP Lett. 48, 536 (1988)].

${ }^{5}$ I. Bratberg, A. Hansen, and E. H. Hauge, Europhys. Lett. 37, 19 (1997).
${ }^{6}$ J. T. Chalker and P. D. Coddington, J. Phys. C 21, 2665 (1988).

${ }^{7}$ L. Jaeger, J. Phys. C 3, 2441 (1991).

${ }^{8}$ R. Klesse and M. Metzler, Europhys. Lett. 32, 229 (1995).

${ }^{9}$ D. H. Lee, Z. Wang, and S. Kivelson, Phys. Rev. Lett. 70, 4130 (1993).

${ }^{10}$ We are grateful to D. H. Lee and S. Kivelson for pointing this out to us.

${ }^{11}$ P. Kratzer and W. Brenig, Z. Phys. B 94, 147 (1994).

${ }^{12}$ B. M. Gammel and W. Brenig, Phys. Rev. B 53, R13 279 (1996).

${ }^{13}$ F. Evers and W. Brenig, Z. Phys. B 94, 155 (1994).

${ }^{14}$ J. F. G. Eastmond, Ph.D. thesis, University of Oxford, 1993.

${ }^{15}$ K. Wysokinski, F. Evers, and W. Brenig, Phys. Rev. B 54, 10720 (1996). 
${ }^{16}$ H. A. Fertig and B. I. Halperin, Phys. Rev. B 36, 7969 (1987).

${ }^{17}$ Recently, R. Klesse and M. Metzler discussed the network model for similar values of $W$, but their primary interest was in its multifractal features (Ref. 8).

${ }^{18}$ Formally, both models can be defined by an appropriate choice of a matrix $\mathbf{M}^{\prime}$. For a definition see the original work by J. T. Chalker and P. D. Coddington (Ref. 6). Model $\mathbf{M}_{\mathbf{s}}$ corresponds to a symmetric arrangement of the minus signs,

$$
\mathbf{M}^{\prime}=\left(\begin{array}{cc}
\sqrt{1+e^{\pi \gamma}} & -\sqrt{e^{\pi \gamma}} \\
-\sqrt{e^{\pi \gamma}} & \sqrt{1+e^{\pi \gamma}}
\end{array}\right),
$$

whereas they are nonsymmetric in model $\mathbf{M}_{\mathbf{a}}$,

$$
\mathbf{M}^{\prime}=\left(\begin{array}{cc}
\sqrt{1+e^{\pi \gamma}} & -\sqrt{e^{\pi \gamma}} \\
\sqrt{e^{\pi \gamma}} & -\sqrt{1+e^{\pi \gamma}}
\end{array}\right) .
$$

${ }^{19}$ D. H. Lee, Phys. Rev. B 50, 7016 (1994).

${ }^{20}$ J. K. Jain and S. Kivelson, Phys. Rev. B 37, 4111 (1987).

${ }^{21}$ At this point it might be of interest to discuss another question related to the results presented in Fig. 3. Why do the models $\mathbf{M}_{\mathbf{s}}$ and $\mathbf{M}_{\mathbf{r}}$ for large disorder in a certain window of sample sizes exhibit the same critical exponent $\nu \approx \frac{7}{3}$ ? In both models, large but finite disorder seems to be irrelevant. Suppose, in addition, that in both models $\xi_{\text {irr }} \propto W^{4 / 3}$ is the signature of classical orbits underlying the quantum-mechanical wave function in the energy regime $\epsilon \gg \Delta$. It seems plausible that, as assumed by Milnikov and Sokolov, $\xi_{p}(\epsilon)$ sets the underlying scale of the problem. As the unperturbed localization length diverges like $\epsilon^{-\nu_{s}}$ in model $\mathbf{M}_{\mathbf{s}}$ we find $\xi(\epsilon) \propto \xi_{p}(\epsilon) \epsilon^{-\nu_{s}}$ and thus $\nu=\nu_{s}+\frac{4}{3}=\frac{7}{3}$ (because $\left.\nu_{s}=1\right)$. We believe that the main difference between the models under investigation is, that $\mathbf{M}_{\mathbf{r}}$ has a tendency to built up large resonances giving rise to the multifractal features (Refs. 28 and 8 ) of the wave function. In contrast, model $\mathbf{M}_{\mathbf{s}}$ does not have this tendency, due to the absence of disorder in the critical limit $W \rightarrow 0$. However, in the classical regime $\epsilon \ll \Delta$, the difference is lifted in both models because a classical substructure sets the underlying length scale.

${ }^{22}$ M. N. Barber, in Phase Transitions and Critical Phenomena 6, edited by C. Domb and J. L. Lebowitz (Academic, New York, 1983).

${ }^{23}$ F. Harris, J. Phys. C 7, 1871 (1974).

${ }^{24}$ R. Klesse, Ph.D. thesis, University of Cologne, 1996.

${ }^{25}$ The fact that $\sigma_{x x}(\omega)$ is proportional to $\omega^{0}$ in the scaling regime can easily be shown analytically in within the semiclassical theory (Ref. 13).

${ }^{26}$ V. T. Dolgopolov et al., Pis'ma Zh. Éksp. Teor. Fiz. 62, 152 (1995); A. A. Shashkin et al., Phys. Rev. Lett. 73, 3141 (1994).

${ }^{27}$ B. Kramer, Phys. Rev. B 47, 9888 (1993).

${ }^{28}$ B. Huckestein and L. Schweitzer, Phys. Rev. Lett. 72, 713 (1994). 